\title{
MODELING AND CONTROL OF A COMPLIANTLY ENGINEERED ANTHROPOMIMETIC ROBOT IN CONTACT TASKS
}

\author{
Veljko Potkonjak \\ Faculty of Electrical Engineering, University of \\ Belgrade, Bulevar kralja Aleksandra 73, 11000 \\ Belgrade, Serbia (corresponding author, tel: +381 \\ 11 3218318; e-mail: potkonjak@yahoo.com)
}

\author{
Kosta Jovanovic \\ Faculty of Electrical Engineering, University of \\ 11000 Belgrade, Bulevar kralja Aleksandra 73, \\ Belgrade, Serbia (e-mail: kostaj@etf.rs)
}

\author{
Bratislav Svetozarevic \\ Automatic Control Laboratory, \\ Swiss Federal Institute of \\ Technology-ETH, Physiksrasse \\ 3, 8092 Zurich, Switzerland \\ (e-mail: \\ bratislavs@control.ee.ethz.ch)
}

\author{
Owen Holland \\ School of Informatics, University \\ of Sussex, Brighton BN19 QJ, \\ Falmer, United Kingdom \\ (e-mail: \\ owen.holland@gmail.com)
}

\author{
Dusan Mikicic \\ Faculty of Electrical \\ Engineering, University of 11000 \\ Belgrade, Bulevar kralja \\ Aleksandra 73, Belgrade, Serbia \\ (e-mail: mikicic@etf.rs)
}

\section{ABSTRACT}

This paper attempts to develop a dynamic model and design a controller for a fully anthropomorphic, compliantly driven robot. To imitate muscles, the robot's joints are actuated by DC motors antagonistically coupled through tendons. To ensure safe interaction with humans in a human-centered environment, the robot exploits passive mechanical compliance, in the form of elastic springs in the tendons. To enable simulation, the paper first derives a mathematical model of the robot's dynamics, starting from the "Flier" approach. The control of the antagonistic drives is based on a biologically inspired puller-and-follower concept where at any instant the puller is responsible for the joint motion while the follower keeps the inactive tendon from slackening. In designing the controller, it was first necessary to use the advanced theory of nonlinear control for dealing with individual joints, and then to apply the theory of robustness in order to extend control to the multi-jointed robot body.

\section{INTRODUCTION}

Anthropomimetics refers to a new kind of robotics which copies the human body as faithfully as possible with the aim of achieving a level of performances (diversity of motions, maneuverability, etc.) comparable with that of a human. This amounts to a return to the origins of robotic science - creating an artificial man [1]. This paper attempts to develop an appropriate dynamic model and then to design a controller for an existing anthropomimetic humanoid - ECCEROBOT ${ }^{1}$.

ECCEROBOT has joints driven by antagonistically coupled DC motors. The two joint motors, the agonist and antagonist, working through tendons, mimic muscles. Since the robot is expected to work in a human centered environment and in the presence of humans, emphasis is given to safe interaction with the surroundings. To achieve this safety, the robot features passive mechanical compliance, which is implemented by placing elastic springs in the tendons.

\footnotetext{
${ }^{1}$ EU FP7 project: „Embodied Cognition in a Compliantly Engineered Robot“ (www.eccerobot.org)
} 
The paper begins by describing the robot's structure and in particular the proposed mechanical models of the joints. Then it develops a complete mathematical model of the system dynamics. The modeling starts from a preexisting abstract model of a humanoid, called the Flier. It relates joint torques with joint motions and contacts with the environment, for any arbitrary robot task. Torques are produced by compliant agonist-antagonist (AA) drives. A complete dynamic model is obtained, relating the joint and motor motions and the contacts with the environment to the input control voltages. Controlling joints driven by AA pairs in the presence of compliance is a challenging problem. We approach the control problem by first analyzing a single-joint system. The main result is a new biologically-inspired and energy efficient puller-follower concept which enables the control of joint motions while at the same time preventing the unloaded tendon from slackening. Later we extend the treatment to a spatial multi-jointed robot, introducing on-line gravity compensation and robustness to compensate for the effects of variable joint inertia and dynamic coupling between joints. While in previous research the nonconstrained robot motion was considered, the current work includes the solution of contact tasks.

\section{ANTHROPOMIMETIC ROBOT STRUCTURE}

The ECCEROBOT project is attempting to create a robot very similar in function to the human body (Fig. 1.a), by replicating its skeleton and its antagonistic tendon drives. With its compliant human-like shape, such a mechanism is able to interact with humans and with its environment in an inherently safe way.
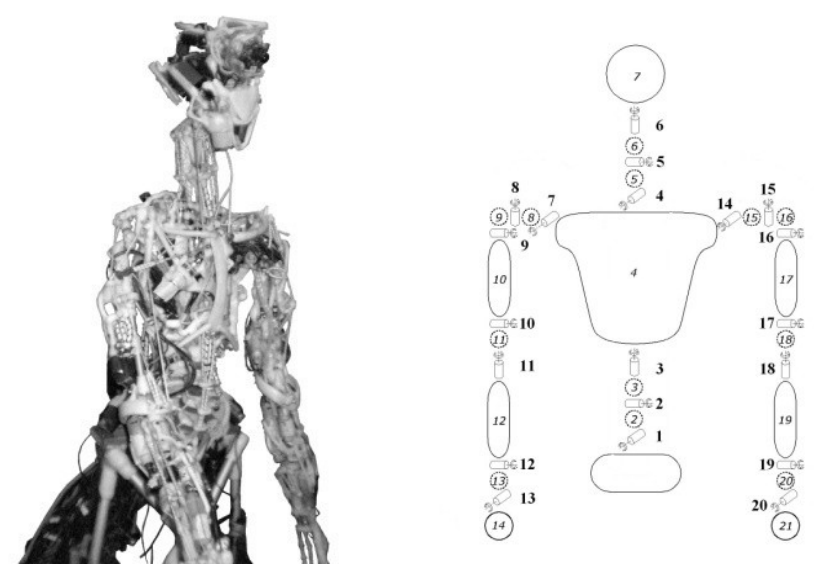

Fig. 1. (a) REAL ROBOT STRUCTURE - ECCEROBOT (b) ROBOT MODEL STRUCTURE

The mechanical complexity and human-like nature of ECCEROBOT is constantly under development. This paper describes its structure using the mechanical model shown in Fig. 1.b [2]. Each joint rotation is driven by two motors working in AA mode. For the elbow rotation, the mechanical model of Fig. 2.a is adopted (the "triangular model"). Motor " $a$ " plays the role of the biceps (or brachialis) while motor " $b$ " mimics the triceps. For the shoulder rotations and other rotations in the robot's body, the mechanical model of Fig. 2.b is used (the "circular model").
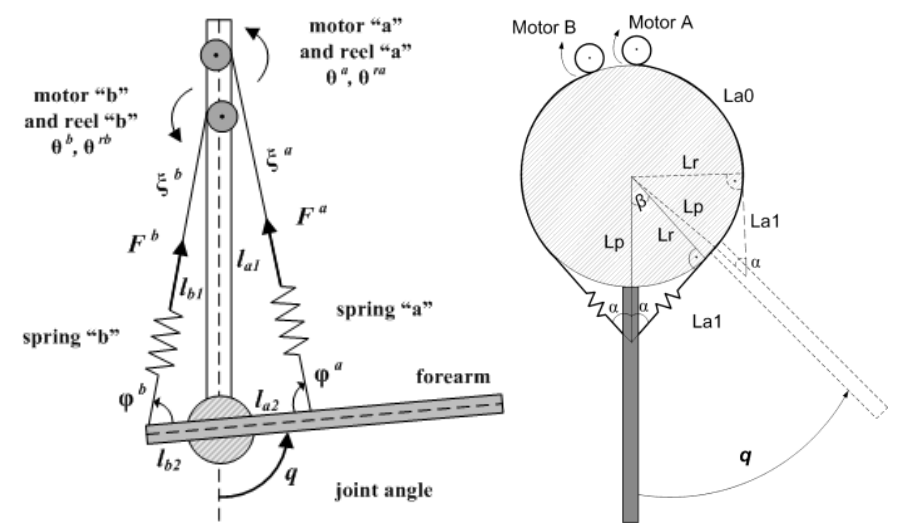

Fig. 2. (a) ELBOW ROTATION WITH AA DRIVES - TRIANGULAR MODEL

(b) SHOULDER ROTATION AA DRIVES - CIRCULAR MODEL

\section{Single-joint drive model}

The geometry of a triangular joint (Fig. 2.a) is defined by

$$
\begin{aligned}
\xi^{a}(q) & =\sqrt{l_{a 1}^{2}+l_{a 2}^{2}+2 l_{a 1} l_{a 2} \cos (q)} \\
\xi^{b}(q) & =\sqrt{l_{b 1}^{2}+l_{b 2}^{2}-2 l_{b 1} l_{b 2} \cos (q)} \\
\varphi^{a} & =\arcsin \left(\frac{l_{a 1} \sin (q)}{\xi^{a}(q)}\right) \\
\varphi^{b} & =\arcsin \left(\frac{l_{b 1} \sin (q)}{\xi^{b}(q)}\right)
\end{aligned}
$$

and for a circular model (Fig. 2.b) it is:

$$
\begin{aligned}
& \xi^{a}(q)=\left(\frac{\pi}{2}+\alpha-q\right) l_{r}+\sqrt{l_{p}^{2}-l_{r}^{2}} \\
& \xi^{b}(q)=\left(\frac{\pi}{2}+\alpha+q\right) l_{r}+\sqrt{l_{p}^{2}-l_{r}^{2}}
\end{aligned}
$$

The elastic (tension) forces in the tendons (cords) have two components each, one proportional (pure elastic deformation) and the other differential (damping). The relationships that describe this are:

$$
\begin{gathered}
F^{a}=k^{a} \Delta l^{a}+d^{a} \Delta \dot{l}^{a} \\
F^{b}=k^{b} \Delta l^{b}+d^{b} \Delta \dot{l}^{b}
\end{gathered}
$$


where $\Delta l^{a}$ and $\Delta l^{b}$ are the linear deformations (cord extensions) equal to:

$$
\begin{aligned}
\Delta l^{a} & =\xi^{a}(q)-\xi_{0}^{a}+r^{a} \theta^{r a} \\
\Delta l^{b} & =\xi^{b}(q)-\xi_{0}^{b}-r^{b} \theta^{r b}
\end{aligned}
$$

where $\xi_{0}^{a(b)}$ is the initial length of cord "a" ("b").

The equations of DC motor dynamics (6) and the relationships for the gear-boxes and pulleys (7) apply to both agonist "a" and antagonist " $b$ " in the same form, therefore, the superscripts $a$ and $b$ are omitted:

$$
\begin{gathered}
C^{M} i=I^{r o t} \ddot{\theta}+B \dot{\theta}+M ; \quad u=R i+C^{E} \dot{\theta} \\
\theta^{r}=\frac{\theta}{N} ; \quad M^{r}=\mu N M ; \quad M^{r}=F r
\end{gathered}
$$

where $C^{M}$ is the torque constant, $I^{\text {rot }}$ is the rotor moment of inertia, $B$ is the viscous friction coefficient, $M$ is the motor output torque, $u$ is the input voltage, $R$ is the armature resistance, $i$ is the motor current, $C^{E}$ is the coefficient of back e.m.f., $\theta$ is the angle of motor shaft rotation, $N$ is the gear-box ratio, $\mu$ is the gear-box efficiency coefficient, $r$ is the reel radius, $\theta^{r}$ is the reel angle (gear-box output angle), $M^{r}$ is the gear-box output torque and $F$ is the tension force in the cord (tendon force).

The tendon forces create a torque $\tau$ about the joint axis: expression (8) holds for the triangular and (9) for the circular joint:

$$
\begin{gathered}
\tau=F^{a} l_{a 2} \sin \left(\varphi^{a}\right)-F^{b} l_{b 2} \sin \left(\varphi^{b}\right) \\
\tau=F^{a} l_{p} \sin (\alpha)-F^{b} l_{p} \sin (\alpha)
\end{gathered}
$$

If the system consisted of one joint only, this torque would move one link (see Fig. 2.), solving its inertia ( $I \ddot{q})$ and the gravity load $\left(M^{g}\right)$ :

$$
\tau=I \ddot{q}+M^{g}(q)
$$

where $I$ is the constant moment of inertia and the gravity term depends on the position $q$.

With the multi-link system, the drive-to-motion relation is much more complex.

\section{The full multi-joint multi-link model}

Here, we derive the dynamic model of the complete body mechanism and integrate it with the joint-drive models defined by (1)-(9).

We start from the "classical" dynamic model that considers the joint torques as the controls and relates them to joint motions. The concept of the Flier approach, derived for humanoid robots, is then applied [3,4]. It originally dealt with a full humanoid (pelvis, torso, arms, legs, head). ECCEROBOT applies only a part of it to the upper-body robot of Fig. 1. The idea of the Flier approach is to consider the humanoid freely flying in space and then to introduce contacts with environmental objects in order to model the imposed motion task. This applies to any motion task: walking and running, manipulation, sporting motions, etc. When applied to the ECCEROBOT structure (Fig. 1.b), the system configuration becomes as shown in Fig. 3.

Following the Flier concept, we start the dynamic analysis from the free-flying model. If robot has $n$ single-rotation joints ( $n=20$ for the configuration under investigation), then its position is defined by a vector of dimension $N=6+n$ :

$$
\mathbf{Q}_{N \times 1}=\left(x, y, z, \delta, \varphi, \psi, q_{1}, \ldots, q_{n}\right)=\left(\mathbf{X}_{6 \times 1}, \mathbf{q}_{n \times 1}\right)
$$

where $\mathbf{X}=(x, y, z, \delta, \varphi, \psi)$ defines the absolute position of the "main body", the pelvis in this case, while $\mathbf{q}=\left(q_{1}, \ldots, q_{n}\right)$ represent joint angles.

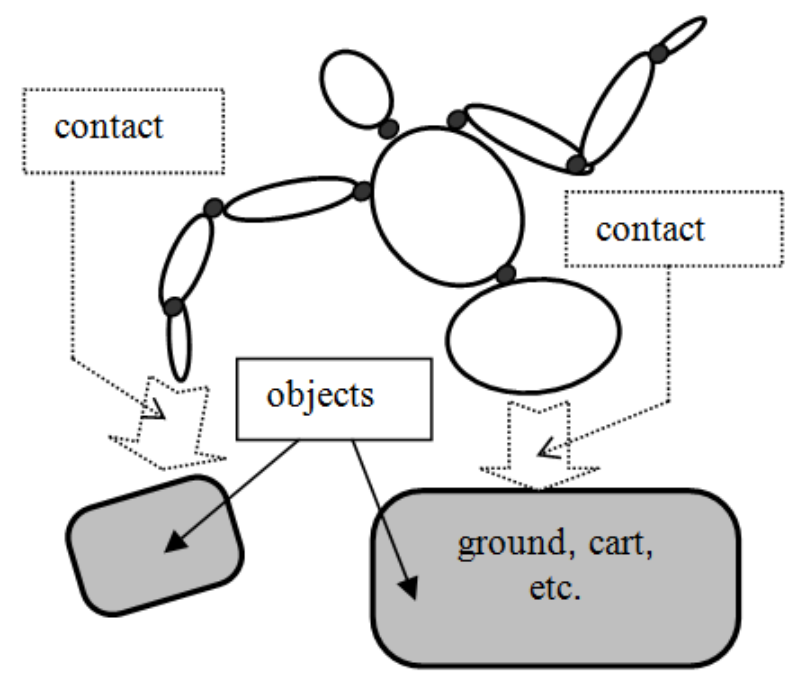

Fig. 3. THE SYSTEM CONFIGURATION

The dynamic model has the matrix form:

$$
\mathbf{H}(\mathbf{Q}) \ddot{\mathbf{Q}}+\mathbf{h}(\mathbf{Q}, \dot{\mathbf{Q}})=\mathbf{T}
$$

where $\quad \mathbf{T}_{N \times 1}=\left(0, \ldots, 0, \tau_{1}, \ldots, \tau_{n}\right)=\left(\mathbf{0}_{6 \times 1}, \boldsymbol{\tau}_{n \times 1}\right) \quad$ is $\quad$ the generalized vector of drives, $\boldsymbol{\tau}=\left(\tau_{1}, \ldots, \tau_{n}\right)$ represents the 
joint torques, $\mathbf{H}_{N \times N}$ is the inertial matrix, and $\mathbf{h}_{N \times 1}$ takes care of gravity, centrifugal, and Coriolis' effects.

The contacts (one or more) with the environment are now introduced. Contact refers to a particular robot link and restricts the relative motion of that link with respect to the relevant environmental object. If there are $m$ restricted directions, the contact can be expressed as:

$$
\mathbf{s}^{\mathbf{c}}\left(\mathbf{Q}, \mathbf{Q}_{\mathrm{b}}\right)=\mathbf{0}
$$

where $\mathbf{s}_{m \times 1}^{\mathbf{c}}$ is the vector of relative link-to-object position, which depends on the robot position $\mathbf{Q}$ (of dimension $N$ ) and the object position $\mathbf{Q}_{\mathrm{b}}$ (of dimension $k$ ). By derivation:

$$
\begin{gathered}
\dot{\mathbf{s}}^{\mathbf{c}}=\mathbf{J}\left(\mathbf{Q}, \mathbf{Q}_{\mathrm{b}}\right) \dot{\mathbf{Q}}+\mathbf{J}_{\mathbf{b}}\left(\mathbf{Q}, \mathbf{Q}_{\mathrm{b}}\right) \dot{\mathbf{Q}}_{\mathbf{b}}=\mathbf{0} \\
\ddot{\mathbf{s}}^{\mathbf{c}}=\mathbf{J}\left(\mathbf{Q}, \mathbf{Q}_{\mathrm{b}}\right) \ddot{\mathbf{Q}}+\mathbf{J}_{\mathbf{b}}\left(\mathbf{Q}, \mathbf{Q}_{\mathrm{b}}\right) \ddot{\mathbf{Q}}_{\mathbf{b}}+\mathbf{A}\left(\mathbf{Q}, \dot{\mathbf{Q}}, \mathbf{Q}_{\mathbf{b}}, \dot{\mathbf{Q}}_{\mathrm{b}}\right)=\mathbf{0}
\end{gathered}
$$

where $\mathbf{J}=\frac{\partial \mathbf{s}^{\mathbf{c}}}{\partial \mathbf{Q}}$ and $\mathbf{J}_{\mathbf{b}}=\frac{\partial \mathbf{s}^{\mathbf{c}}}{\partial \mathbf{Q}_{\mathbf{b}}}$ are Jacobeans of dimensions $m \times N$ and $m \times k$ respectively, and $\mathbf{A}$ contains second partial derivatives.

Contact introduces reaction forces and moments. Reactions appear along the restricted directions $\mathbf{s}^{\mathbf{c}}$. Let $\mathbf{R}_{m \times 1}$ be the vector of reactions.

The dynamics of the contact motion is now described by the model:

$$
\begin{gathered}
\mathbf{H}(\mathbf{Q}) \ddot{\mathbf{Q}}+\mathbf{h}(\mathbf{Q}, \dot{\mathbf{Q}})=\mathbf{T}+\mathbf{J}^{\mathbf{T}}\left(\mathbf{Q}, \mathbf{Q}_{\mathbf{b}}\right) \mathbf{R} \\
\ddot{\mathbf{s}}^{\mathbf{c}}=\mathbf{J}\left(\mathbf{Q}, \mathbf{Q}_{\mathrm{b}}\right) \ddot{\mathbf{Q}}+\mathbf{J}_{\mathbf{b}}\left(\mathbf{Q}, \mathbf{Q}_{\mathrm{b}}\right) \ddot{\mathbf{Q}}_{\mathbf{b}}+\mathbf{A}\left(\mathbf{Q}, \dot{\mathbf{Q}}, \mathbf{Q}_{\mathbf{b}}, \dot{\mathbf{Q}}_{\mathbf{b}}\right)=\mathbf{0}(15 \mathrm{~b}) \\
\mathbf{W}\left(\mathbf{Q}_{\mathbf{b}}\right) \ddot{\mathbf{Q}}_{\mathrm{b}}+\mathbf{w}\left(\mathbf{Q}_{\mathbf{b}}, \dot{\mathbf{Q}}_{\mathrm{b}}\right)=\mathbf{T}_{\mathbf{b}}-\mathbf{J}_{\mathbf{b}} \mathbf{T}\left(\mathbf{Q}, \mathbf{Q}_{\mathbf{b}}\right) \mathbf{R}
\end{gathered}
$$

The model unites the robot dynamics ( $N$-dimensional submodel (15a)), the object dynamics ( $k$-dimensional submodel (15c) with model matrices $\mathbf{W}_{k \times k}$ and $\mathbf{w}_{k \times 1}$ ), and the geometry of contact ( $m$-dimensional subsystem (15b)). It relates the driving torques ( $\mathbf{T}$ for the robot and $\mathbf{T}_{\mathrm{b}}$ for the object) to the motion ( $\mathbf{Q}$ and $\mathbf{Q}_{b}$ for the robot and the object respectively) and the contact reactions (R). If the behavior of the contacted object is robust to the robot actions (e.g. if the object is massive, like the ground), then the robot dynamics can be decoupled and the (15a) and (15b) can be solved considering the object motion to be zero.

Bearing in mind the previously derived equations of the joint drives (relations (8), (9), and (1)-(5)) it is clear that each joint torque can be written as a function of the joint and motor positions $\left(q, \theta^{a}, \theta^{b}\right)$ and velocities $\left(\dot{q}, \dot{\theta}^{a}, \dot{\theta}^{b}\right): \tau_{j}=$ $\tau_{j}\left(q_{j}, \dot{q}_{j}, \theta^{a}{ }_{j}, \dot{\theta}^{a}{ }_{j}, \theta^{b}{ }_{j}, \dot{\theta}^{b}{ }_{j}\right)$ and so in matrix form

$$
\mathbf{T}_{N \times 1}=\mathbf{T}\left(\mathbf{q}, \dot{\mathbf{q}}, \boldsymbol{\theta}^{\mathbf{a}}, \dot{\boldsymbol{\theta}}^{\mathbf{a}}, \boldsymbol{\theta}^{\mathbf{b}}, \dot{\boldsymbol{\theta}}^{\mathbf{b}}\right)
$$

where $\mathbf{q}, \boldsymbol{\theta}^{\mathbf{a}}, \boldsymbol{\theta}^{\mathbf{b}}$ are proper position vectors (for joints and motors). Note that the compliance in the tendons makes the motor angles independent of joint position and thus increases the dimensionality of the problem.

Substituting $\mathbf{T}$ in (15a), one obtains:

$$
\mathbf{H}(\mathbf{Q}) \ddot{\mathbf{Q}}+\tilde{\mathbf{h}}\left(\mathbf{Q}, \dot{\mathbf{Q}}, \boldsymbol{\theta}^{\mathbf{a}}, \dot{\boldsymbol{\theta}}^{\mathbf{a}}, \boldsymbol{\theta}^{\mathrm{b}}, \dot{\boldsymbol{\theta}}^{\mathrm{b}}\right)=\mathbf{J}^{\mathrm{T}}\left(\mathbf{Q}, \mathbf{Q}_{\mathbf{b}}\right) \mathbf{R}
$$

where $\quad \tilde{\mathbf{h}}_{N \times 1}\left(\mathbf{Q}, \dot{\mathbf{Q}}, \boldsymbol{\theta}^{\mathbf{a}}, \dot{\boldsymbol{\theta}}^{\mathbf{a}}, \boldsymbol{\theta}^{\mathbf{b}}, \dot{\boldsymbol{\theta}}^{\mathbf{b}}\right)=\mathbf{h}_{N \times 1}(\mathbf{Q}, \dot{\mathbf{Q}})-$ $\mathbf{T}_{N \times 1}\left(\mathbf{Q}, \dot{\mathbf{Q}}, \boldsymbol{\theta}^{\mathbf{a}}, \dot{\boldsymbol{\theta}}^{\mathbf{a}}, \boldsymbol{\theta}^{\mathbf{b}}, \dot{\boldsymbol{\theta}}^{\mathbf{b}}\right)$. This relation replaces (15a) in model (15).

The dynamics of the motors ((6)-(7) along with (1)-(5), applied to each joint $j=1, \ldots, n)$ can be rewritten to give an additional $2 n$ differential equations in the following matrix $2 n$ dimensional form:

$$
\overline{\overline{\mathbf{H}}} \ddot{\boldsymbol{\theta}}+\overline{\overline{\mathbf{h}}}\left(\mathbf{Q}, \dot{\mathbf{Q}}, \boldsymbol{\theta}^{\mathbf{a}}, \dot{\boldsymbol{\theta}}^{\mathbf{a}}, \boldsymbol{\theta}^{\mathbf{b}}, \dot{\boldsymbol{\theta}}^{\mathbf{b}}\right)=\mathbf{C} \mathbf{u}
$$

where $\boldsymbol{\theta}=\left(\boldsymbol{\theta}^{\mathbf{a}}, \boldsymbol{\theta}^{\mathbf{b}}\right)$ and $\mathbf{u}_{2 n \times 1}$ is the vector of input control voltages. Model matrices $\overline{\overline{\mathbf{H}}}$ and $\overline{\overline{\mathbf{h}}}$ are derived starting from (1)-(7).

Thus, model (15), with (17) substituted, describes the contact dynamics of the robot body and (18) describes the dynamics of the robot motors. We now join these relationships, obtaining a set of $N+m+k+2 n$ scalar equations:

$$
\begin{gathered}
\mathbf{H}(\mathbf{Q}) \ddot{\mathbf{Q}}+\tilde{\mathbf{h}}\left(\mathbf{Q}, \dot{\mathbf{Q}}, \boldsymbol{\theta}^{\mathbf{a}}, \dot{\boldsymbol{\theta}}^{\mathrm{a}}, \boldsymbol{\theta}^{\mathrm{b}}, \dot{\boldsymbol{\theta}}^{\mathrm{b}}\right)=\mathbf{J}^{\mathrm{T}}\left(\mathbf{Q}, \mathbf{Q}_{\mathbf{b}}\right) \mathbf{R} \\
\ddot{\mathbf{s}}^{\mathrm{c}}=\mathbf{J}\left(\mathbf{Q}, \mathbf{Q}_{\mathrm{b}}\right) \ddot{\mathbf{Q}}+\mathbf{J}_{\mathbf{b}}\left(\mathbf{Q}, \mathbf{Q}_{\mathrm{b}}\right) \ddot{\mathbf{Q}}_{\mathrm{b}}+\mathbf{A}\left(\mathbf{Q}, \dot{\mathbf{Q}}, \mathbf{Q}_{\mathbf{b}}, \dot{\mathbf{Q}}_{\mathrm{b}}\right)=\mathbf{0} \\
\mathbf{W}\left(\mathbf{Q}_{\mathrm{b}}\right) \ddot{\mathbf{Q}}_{\mathrm{b}}+\mathbf{w}\left(\mathbf{Q}_{\mathbf{b}}, \dot{\mathbf{Q}}_{\mathrm{b}}\right)=\mathbf{T}_{\mathbf{b}}-\mathbf{J}_{\mathbf{b}}{ }^{\mathrm{T}}\left(\mathbf{Q}, \mathbf{Q}_{\mathbf{b}}\right) \mathbf{R} \\
\overline{\overline{\mathbf{H}}} \ddot{\boldsymbol{\theta}}+\overline{\overline{\mathbf{h}}}\left(\mathbf{Q}, \dot{\mathbf{Q}}, \boldsymbol{\theta}^{\mathbf{a}}, \dot{\boldsymbol{\theta}}^{\mathrm{a}}, \boldsymbol{\theta}^{\mathrm{b}}, \dot{\boldsymbol{\theta}}^{\mathrm{b}}\right)=\mathbf{C} \mathbf{u}
\end{gathered}
$$

If control $\mathbf{u}$ is given, system (19) can be solved for $\mathrm{N}+2 \mathrm{n}+\mathrm{k}+m$ unknowns: the robot motion $\mathbf{Q}_{N \times 1}$, the motor angles $\left(\boldsymbol{\theta}^{\mathrm{a}}, \boldsymbol{\theta}^{\mathrm{b}}\right)_{2 n \times 1}$, the object motion $\mathbf{Q}_{k \times 1}$, and the contact reactions $\mathbf{R}_{m \times 1}$, thus providing a simulation.

\section{Impact}

Any contact can be regarded as consisting of three phases: the approach, the impact, and regular contact motion. While the approach has been described by the Flier model (12), and the regular contact motion by model (19), the impact needs additional explanation. Although the original Flier concept allows the analysis of rigid vs. soft, and instantaneous vs. 
durable contacts, we here restrict our consideration to the infinitely short impact that happens when two rigid bodies establish a contact which then lasts for some finite time. Such an impact results in infinitely high reaction forces $\mathbf{R}$ but due to the infinitely short time $\Delta \mathrm{t}$ the momentum $\mathbf{R} \Delta \mathrm{t}$ is not infinite. The momentum and the resulting change in system velocities can be calculated from the impact model. The impact model is obtained if the contact model is integrated over the impact period $\Delta t=\left(t^{\prime}, t^{\prime \prime}\right) \rightarrow 0$. Integration yields:

$$
\begin{gathered}
\mathbf{H}(\mathbf{Q}) \Delta \dot{\mathbf{Q}}=\mathbf{J}^{\mathrm{T}}\left(\mathbf{Q}, \mathbf{Q}_{\mathbf{b}}\right) \mathbf{R} \Delta t \\
\mathbf{J}\left(\mathbf{Q}, \mathbf{Q}_{\mathbf{b}}\right) \Delta \dot{\mathbf{Q}}+\mathbf{J}_{\mathbf{b}}\left(\mathbf{Q}, \mathbf{Q}_{\mathbf{b}}\right) \Delta \dot{\mathbf{Q}}_{\mathbf{b}}=\dot{\mathbf{s}}^{\mathbf{c}}\left(\mathrm{t}^{\prime}\right) \\
\mathbf{W}\left(\mathbf{Q}_{\mathbf{b}}\right) \Delta \dot{\mathbf{Q}}_{\mathbf{b}}=-\mathbf{J}_{\mathbf{b}}{ }^{\mathrm{T}}\left(\mathbf{Q}, \mathbf{Q}_{\mathbf{b}}\right) \mathbf{R} \Delta t \\
\overline{\overline{\mathbf{H}}} \Delta \dot{\boldsymbol{\theta}}=\mathbf{0}
\end{gathered}
$$

The impact model can then be solved for the change in velocities: $\Delta \dot{\mathbf{Q}}, \Delta \dot{\mathbf{Q}}_{\mathbf{b}}, \Delta \dot{\boldsymbol{\theta}}$ and the impact momentum $\mathbf{R} \Delta t$.

In this paper the robot will establish two contacts with its environment. One contact is between the pelvis and the ground, and the other results from catching a ball (a hand-ball contact).

\section{CONTROL SYNTHESIS FOR AN ISOLATED JOINT}

In this Section we propose a new control approach for an electrical AA drive and present an application of this approach to the joints shown in Fig. 2.a and Fig. 2.b.

The main problem of controlling an electrical AA drive lies in the antagonistic drive's redundancy. One motor, the agonist, initiates the action, while the other, the antagonist, opposes it. In order to achieve a desired joint motion, the motors should be driven simultaneously, in a coordinated way. We also have to consider the tendons coupling the motors to the joint. This tendon coupling enforces unidirectional power transmission and, without the correct control, the tendons can slacken, leading to an undesirable backlash in the torque transmission. These two problems were initially addressed in $[5,6]$ and several control strategies were proposed in [6]. Further analysis of this type of mechanism was presented in [7]. Biologically-inspired approaches were developed in [8,9]. Since all the proposed concepts used linearly coupled drives, they were inapplicable to our nonlinearly coupled system. In order to deal with this complex control problem, we developed a completely new biologically-inspired and energy efficient approach - the puller-follower concept $[2,10]$. As our main reference for human physiology we used [11]. We now briefly present this new approach. Due to lack of space, the energy efficiency will not be proved exactly. This energy statement will hopefully be acceptable after describing the concept which avoids unnecessary high tensions in tendons.

Let us first describe our control concept by starting with the main control requirements for an isolated joint. A generalization to a multi-joint multi-link system will then be made. The basic control requirement is the control of the joint position. One motor, the puller, takes the main responsibility for this task. In order to prevent slackening of the other tendon, a second control task - maintaining an appropriate tension in the tendon - is added. This additional task is mainly assigned to the follower motor. By "appropriate tension" we mean a tension above some minimal value that guarantees that a tendon will remain stretched during joint motion. This prescribed minimum will be called the reference tension force. Accordingly, in each joint we will have two references: the reference position (i.e. the reference motion trajectory) and the reference tension force. The joint motors exchange roles when the motion requires it, usually when acceleration turns to deceleration. This exchange of roles is called switching.

We now design the joint control based on the pullerfollower concept. The state-space coordinates are adopted as follows: motor angles and angular velocities, and joint angle and velocity: $x_{1}=\theta^{a}, x_{2}=\dot{\theta}^{a}, x_{3}=\theta^{b}, x_{4}=\dot{\theta}^{b}$, $x_{5}=q, x_{6}=\dot{q}$. Starting from the equations of motors and tendon transmission, (1)-(10), applied to an isolated joint moving in horizontal plane, one derives the canonical model:

$$
\begin{gathered}
\dot{x}_{1}=x_{2} \\
\dot{x}_{2}=x_{2 p}\left(x_{1}, x_{2}, x_{5}, x_{6}\right)+\left(C^{M a} / R^{a} I^{\text {rot } a}\right) u^{a} \\
\dot{x}_{3}=x_{4} \\
\dot{x}_{4}=x_{4 p}\left(x_{3}, x_{4}, x_{5}, x_{6}\right)+\left(C^{M b} / R^{b} I^{r o t} b\right) u^{b} \\
\dot{x}_{5}=x_{6} \\
\dot{x}_{6}=x_{6 p}\left(x_{1}, x_{2}, x_{3}, x_{4}, x_{5}, x_{6}\right)
\end{gathered}
$$

Where the functions of the state $x_{2 p}, x_{4 p}$, and $x_{6 p}$ are nonlinear (for a triangular joint) or linear (for a circular joint). For a triangular model the functions are:

$$
\begin{gathered}
x_{2 p}\left(x_{1}, x_{2}, x_{5}, x_{6}\right)= \\
-\frac{r^{a^{2}} k^{a}}{\mu^{a} N^{a^{2}} I^{\text {rot } a}} x_{1}-\frac{1}{I^{\text {rot } a}}\left(\frac{r^{a^{2}} d^{a}}{\mu^{a} N^{a^{2}}}+\left(B^{a}+\frac{C^{M a} C^{E a}}{R^{a}}\right)\right) x_{2} \\
+\frac{r^{a}}{\mu^{a} N^{a} I^{\text {rot } a}}\left(-k^{a} \xi^{a}\left(x_{5}\right)+\frac{d^{a} l_{a 1} l_{a 2} \sin \left(x_{5}\right)}{\xi^{a}\left(x_{5}\right)} x_{6}+k^{a} \xi_{0}^{a}\right) \\
x_{4 p}\left(x_{3}, x_{4}, x_{5}, x_{6}\right)= \\
-\frac{r^{b^{2}} k^{b}}{\mu^{b} N^{b^{2}} I^{\text {rot b }}} x_{3}-\frac{1}{I^{\text {rot } b}}\left(\frac{r^{b^{2}} d^{b}}{\mu^{b} N^{b^{2}}}+\left(B^{b}+\frac{C^{M b} C^{E b}}{R^{b}}\right)\right) x_{4} \\
+\frac{r^{b}}{\mu^{b} N^{b} I^{\text {rot } b}}\left(k^{b} \xi^{b}\left(x_{5}\right)+\frac{d^{b} l_{b 1} l_{b 2} \sin \left(x_{5}\right)}{\xi^{b}\left(x_{5}\right)} x_{6}-k^{b} \xi_{0}^{b}\right) \\
x_{6 p}\left(x_{1}, x_{2}, x_{3}, x_{4}, x_{5}, x_{6}\right)=
\end{gathered}
$$




$$
\begin{gathered}
\frac{C_{S a}\left(x_{5}\right) r^{a} k^{a}}{N^{a} I} x_{1}+\frac{C_{S a}\left(x_{5}\right) r^{a} d^{a}}{N^{a} I} x_{2}+\frac{C_{S b}\left(x_{5}\right) r^{b} k^{b}}{N^{b} I} x_{3} \\
+\frac{C_{S b}\left(x_{5}\right) r^{b} d^{b}}{N^{b} I} x_{4}+\frac{1}{I} C_{S a}\left(x_{5}\right) k^{a} \xi^{a}\left(x_{5}\right)-\frac{1}{I} C_{S b}\left(x_{5}\right) k^{b} \xi^{b}\left(x_{5}\right) \\
\quad-\frac{1}{I} C_{S a}\left(x_{5}\right) k^{a} \xi_{0}^{a}+\frac{1}{I} C_{S b}\left(x_{5}\right) k^{b} \xi_{0}^{b} \\
-\frac{1}{I}\left(\frac{C_{S a}\left(x_{5}\right) d^{a} l_{a 1} l_{a 2}}{\xi^{a}\left(x_{5}\right)}+\frac{C_{S b}\left(x_{5}\right) b^{b} l_{b 1} l_{b 2}}{\xi^{b}\left(x_{5}\right)}\right) \sin x_{5} \cdot x_{6}
\end{gathered}
$$

where $I$ is the moment of inertia of the link, and $C_{S a}\left(x_{5}\right)$ and $C_{S b}\left(x_{5}\right)$ are functions introduced to simplify the above expression; they are defined as:

$$
C_{S a}\left(x_{5}\right)=\frac{l_{a 1} l_{a 2} \sin x_{5}}{\xi^{a}\left(x_{5}\right)}, C_{S b}\left(x_{5}\right)=\frac{l_{b 1} l_{b 2} \sin x_{5}}{\xi^{b}\left(x_{5}\right)}
$$

For the circular model, it holds that:

$$
\begin{gathered}
x_{2 p}\left(x_{1}, x_{2}, x_{5}, x_{6}\right)= \\
-\frac{r^{a^{2}} k^{a}}{\mu^{a} N^{a^{2}} I^{\text {rot } a}} x_{1}-\frac{1}{I^{\text {rot } a}}\left(\frac{r^{a^{2}} d^{a}}{\mu^{a} N^{a^{2}}}+\left(B^{a}+\frac{C^{M a} C^{E a}}{R^{a}}\right)\right) x_{2} \\
-\frac{r^{a} k^{a}}{\mu^{a} N^{a} I^{\text {rot } a}}\left(\xi^{a}\left(x_{5}\right)-\xi_{0}^{a}\right)+\frac{r^{a} d^{a} l_{r}}{\mu^{a} N^{a} I^{\text {rot a }}} x_{6}
\end{gathered}
$$

$$
\begin{aligned}
& x_{4 p}\left(x_{3}, x_{4}, x_{5}, x_{6}\right)= \\
& -\frac{r^{b^{2}} k^{b}}{\mu^{b} N^{b^{2}} I^{\text {rot } b}} x_{3}-\frac{1}{I^{\text {rot } b}}\left(\frac{r^{b^{2}} d^{b}}{\mu^{b} N^{b^{2}}}+\left(B^{b}+\frac{C^{M b} C^{E b}}{R^{b}}\right)\right) x_{4} \\
& +\frac{r^{b} k^{b}}{\mu^{b} N^{b} I^{\text {rot } b}}\left(\xi^{b}\left(x_{5}\right)-\xi_{0}^{b}\right)+\frac{r^{b} d^{b} l_{r}}{\mu^{b} N^{b} r^{\text {rot } b}} x_{6} \\
& x_{6 p}\left(x_{1}, x_{2}, x_{3}, x_{4}, x_{5}, x_{6}\right)= \\
& \frac{l_{r} r^{a} k^{a}}{N^{a} I} x_{1}+\frac{l_{r} r^{a} d^{a}}{N^{a} I} x_{2}+\frac{l_{r} r^{b} k^{b}}{N^{b} I} x_{3}+\frac{l_{r} r^{b} d^{b}}{N^{b} I} x_{4}-\frac{l_{r}^{2}}{I}\left(d^{a}+d^{b}\right) \\
& x_{6}+\frac{l_{r}}{I}\left[k^{a} \xi^{a}\left(x_{5}\right)-k^{b} \xi^{b}\left(x_{5}\right)-k^{a} \xi_{0}^{a}+k^{b} \xi_{0}^{b}\right]
\end{aligned}
$$

In order to transform model (21) into a form where the idea of different motor roles can be applied, we will use a multivariable feedback approach - input-output feedback linearization for MIMO systems [12]. This method counteracts the nonlinearity and interactions in the model and transforms the original two-input-two-output (TITO) system (21) into two decoupled single-input-single-output (SISO) systems. By adopting this approach, we have to solve similar control problems for both joint models. A higher control logic is then applied in the form of four SISO controllers (one position and one force controller for each of the motors) and a switching logic block. The two SISO controllers are active all the time. A complete control scheme for a triangular joint with AA drive is shown in Fig. 4. The same scheme stands for circular-joint model.

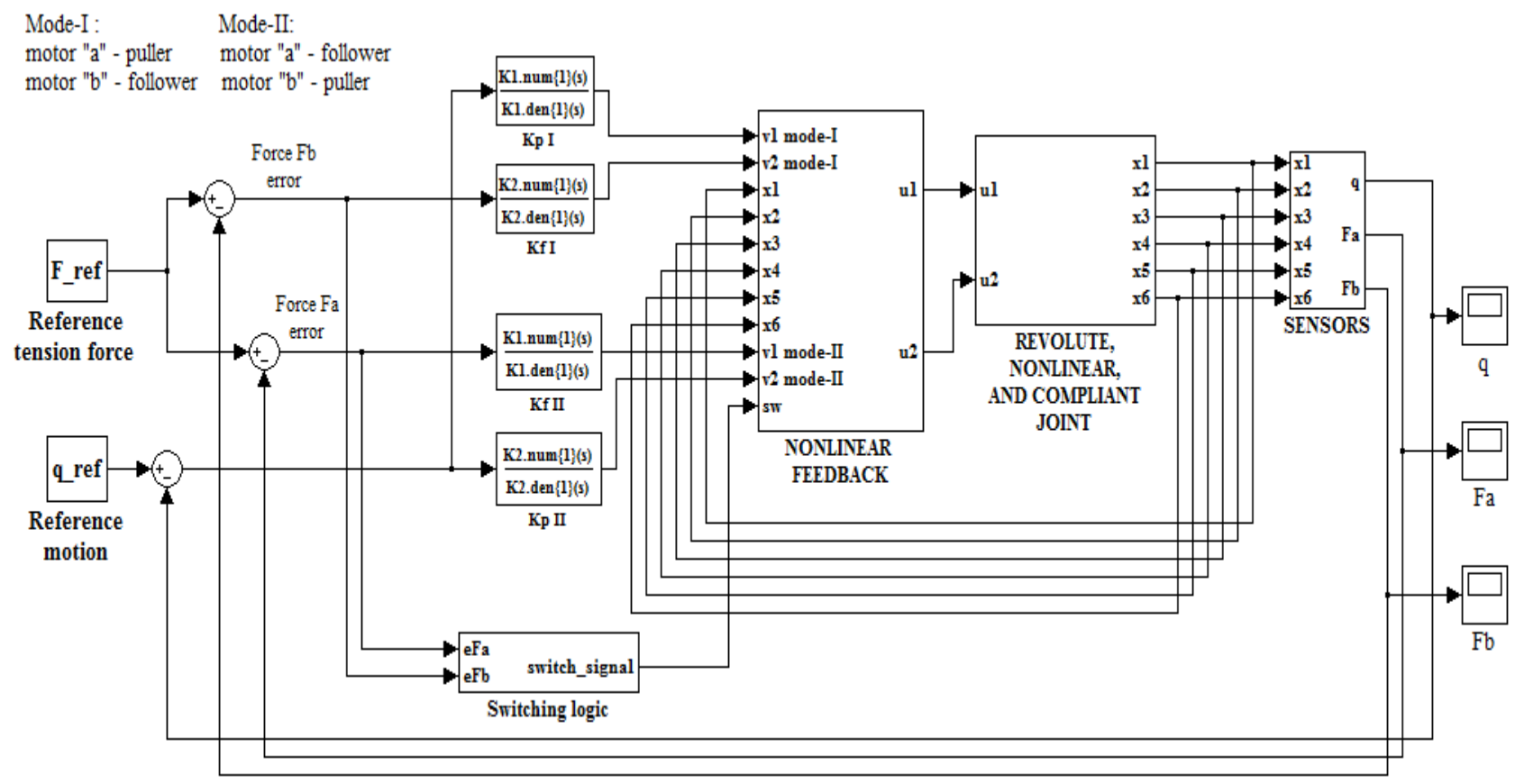

Fig. 4. CONTROL SCHEME FOR THE TRIANGULAR-MODEL JOINT WITH AN ELECTRICAL AA DRIVE APPLIED 
We adopt the same parameters for both motors and therefore it is possible to use the same position and force controllers:

$$
\begin{gathered}
\mathrm{K}_{\mathrm{p}}^{\mathrm{I}}(\mathrm{s})=\mathrm{K}_{\mathrm{p}}^{\mathrm{II}}(\mathrm{s})=\frac{3 \mathrm{e} 7 s^{3}+1.5 \mathrm{e} 8 s^{2}}{s^{3}+3.8 \mathrm{e} 2 s^{2}+4.75 \mathrm{e} 4 s+1.95 \mathrm{e} 6} \\
K_{f}^{I}(s)=K_{f}^{I I}(s)=\frac{3 e 3 s+1.5 e 4}{s+100}
\end{gathered}
$$

The final simulation results are shown in Fig. 5. The position tracks an acceleration-deceleration reference (triangular velocity profile). We can see that the trajectory tracking is good, with a position error of less than $2 \%$, and that exchanging the motor roles (switching) does not compromise the trajectory tracking.

In Fig. 5 one notes that the reference tension force is not kept constant during the motion. The reference tension is increased shortly before switching, and it is reduced after it. The reason for introducing this adaptive reference force comes from the observation that switching often causes a negative overshoot of the tendon force in the transient period after switching.

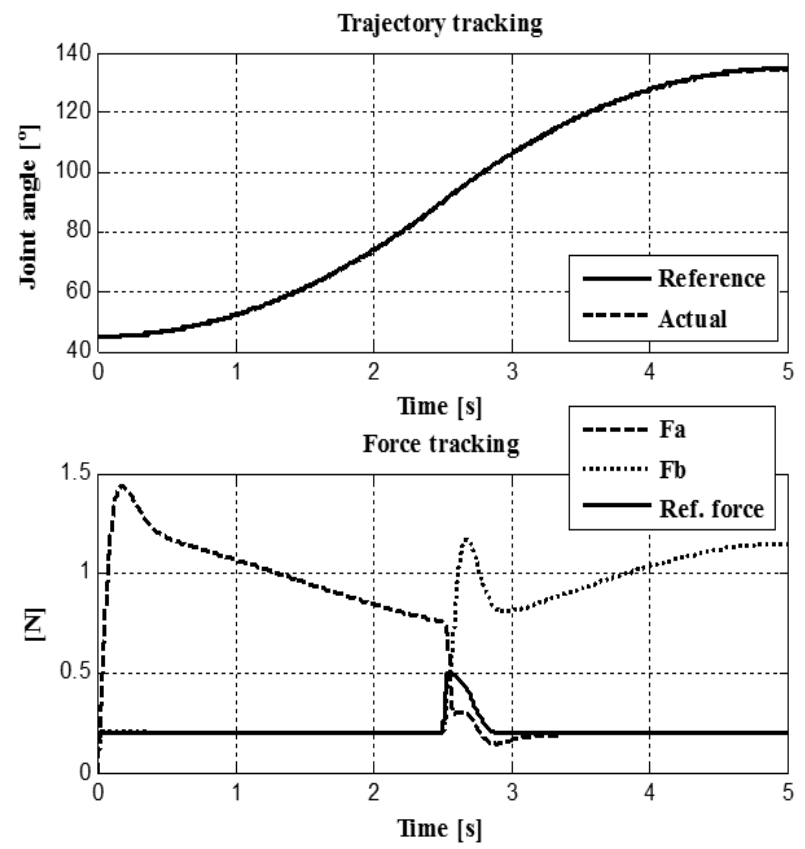

Fig. 5. TRAJECTORY TRACKING AND FORCE TRACKING IN A TRIANGULAR-MODEL JOINT

Although this overshoot is relatively small in magnitude, the small reference value can lead to the pulling force (now becoming the following force) falling below zero, i.e. the tendon becomes slack. We have proved [10] that slackening can be prevented by using a higher value for the reference tension force. To avoid an increase in energy consumption, we raise the force reference shortly before switching and reduce it immediately after the transient oscillations end.

Let us be more precise and about the influence of the reference tension to the system dynamics after switching. Our simulations revealed that the switching shock depends strongly on the level of the reference tension force, as shown by the dotted line in Fig. 6. It can be seen that the minimum shock appears when the reference tension force is about $25 \%$ of the actual pulling force. We also examined the minimum values of the tension forces during switching (shown by the dashed line in Fig. 6). It can be seen that if we raise the reference tension force from $19 \%$ to $40 \%$ of the actual pulling force, then both tension forces will be positive (i.e. both tendons will be taut) all the time. Here we introduce the idea of the maximal tension margin, which is the value of the reference tension force (as a percentage of the actual pulling force) at which the minimum value of the tension forces during switching is highest. This occurs when the reference tension force is close to $32 \%$ of the pulling force. Therefore, we can choose the reference tension in order to gain either of two advantages: the minimal switching shock (with the reference tension set to $25 \%$ of the actual pulling force) or the maximal tension margin (with the reference tension set to $32 \%$ of the actual pulling force).

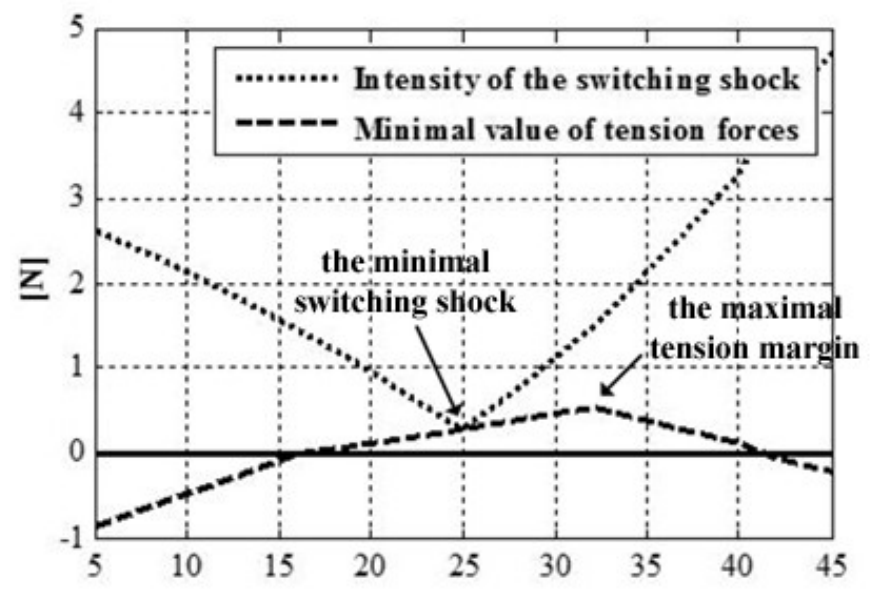

The switching shock as a proportion of the pulling force[\%]

Fig. 6. THE TRADE OFF BETWEEN THE MINIMAL SWITCHING SHOCK AND THE MAXIMAL STRETCHING MARGIN

\section{CONTROL OF THE MULTI-JOINTED ROBOT BODY}

In this Section we consider the complete upper-body model (19). We examine the extent to which the single-joint control strategy can be applied to this multi-joint system. With regard to the joint control the new features are that the joint inertia and gravity load now depend on the positions of other joints, and that dynamic coupling between joints exists. Simulation experiments show that the control derived for isolated joints is 
not satisfactory in the new situation - the nonlinear part of the control system suggested in previous Section cannot completely counteract the new effects. Thus the control performance is compromised.

The solution we propose for this problem is a robust control system design, together with nonlinear compensation for the effects of gravity.

\section{Gravity compensation}

The state space model of the joint in (21) does not consider gravity. The aim of this Section is to include gravity and to define proper compensation.

In order to take care of the fact that the gravity load of a joint depends on the positions of the other joints, we start from the multi-joint system and extract the gravity component from the dynamic model (15a). The vector $\mathbf{h}(\mathbf{Q}, \dot{\mathbf{Q}})$ includes the static gravity load and the dynamic velocity-dependent effects (centrifugal, Coriolis', and viscous friction):

$$
\mathbf{h}(\mathbf{Q}, \dot{\mathbf{Q}})=\mathbf{h}_{g}(\mathbf{Q})+\mathbf{h}_{d}(\mathbf{Q}, \dot{\mathbf{Q}})
$$

To control a particular joint - $j$, we use a single joint notation where the gravity moment is expressed as:

$$
G_{j}=h_{g_{j}}(\mathbf{q})
$$

One of the possible solutions for gravity compensation is to determine the modified feedback linearization for the system including the gravity component. We therefore modify (21):

$$
x_{6 p N E W}\left(x_{1}, x_{2}, x_{3}, x_{4}, x_{5}, x_{6}\right)=x_{6 p}-\frac{1}{I} G_{j}
$$

This changes the corresponding values in the decoupling matrix, thus making the control system aware of the influence of gravity.

\section{Uncertain effective joint inertia and dynamic coupling between joints}

In this Section we show how it is possible to deal with variable joint inertia, and with the effects coming from the dynamic coupling between joints. The proposed solutions are supported by simulation experiments based on the dynamic models (19) and (20).

In contrast to the single-joint systems shown in Fig. 2, where the moment of inertia of a segment is constant and known, in a multi-joint system the effective joint inertia changes and generally depends on the actual position of the entire system. Therefore, from the single-joint viewpoint, the effective inertia represents an uncertain parameter. On the other hand, the coupling between joints represents unmodelled dynamics. In order to deal with the uncertainty and unmodelled dynamics, we designed a robust control system. Because of the applied input-output feedback linearization, the uncertainty in the system cannot be described as a function of system parameters. Therefore we use the $H_{\infty}$ loop-shaping method - a combination of loop shaping and robust stabilization as proposed in [13]. Instead of the existing four SISO controllers (the position and force controllers shown in Fig. 4) we propose four new SISO controllers - two for position and two for force:

$$
\begin{gathered}
\mathrm{K}_{\mathrm{p}}^{\mathrm{I}}(\mathrm{s})=\mathrm{K}_{\mathrm{p}}^{\mathrm{II}}(\mathrm{s})=\frac{9.15 e 9 s^{3}+2.6 e 11 s^{2}+4.2 e 12 s+2.8 e 13}{s^{3}+6.3 e 3 s^{2}+3.9 e 5 s+8.9 e 7} \\
K_{f}^{I}(s)=K_{f}^{I I}(s)=\frac{1.9 e 4 s+7.6 e 5}{s+2.7 e 2}
\end{gathered}
$$

We now have a robust control system with gravity compensation for a single joint. As mentioned above, we will use this single-joint control strategy to control each joint in the multi-joint system. In order to test the robustness of the control system, we introduce an external disturbance in addition to the existing uncertainty and unmodeled dynamics. The simulation experiment consists of raising the arm and grasping a $0.5 \mathrm{~kg}$ ball. The trajectory tracking and the force tracking in the shoulder joint and the elbow joint (joints 9 and 10 respectively in Fig. 1.b) during this grasping experiment are shown in Fig. 7. The control system shows acceptable behavior: the influence of the external disturbance is reduced after $1 s$ to a position error of less than $2 \%$; steady state position errors do not exist; and the tendons are taut all the time, even though the reference tension force is set to the very low value of $3 \mathrm{~N}$.

\section{CONCLUSION}

The paper deals with the complex problem of modeling and controlling an anthropomimetic robot. The complexity of this system is caused mainly by its drives which involve two antagonistically working motors per joint, coupled by tendons with passive compliance. These drives make the motor motions independent of the joint motions, thus trebling the total number of degrees of freedom. This redundancy is successfully resolved by separating the roles of the two joint motors, one being the puller responsible for the joint motion, and the other acting as the follower, keeping the inactive tendon from slackening. An adaptive force reference is needed to solve the problem of switching roles between motors, and to provide an energetically efficient control algorithm. This control study started from an isolated joint where the multivariable feedback approach - input-output feedback linearization for MIMO systems - was successfully applied. With a multi-jointed robot 

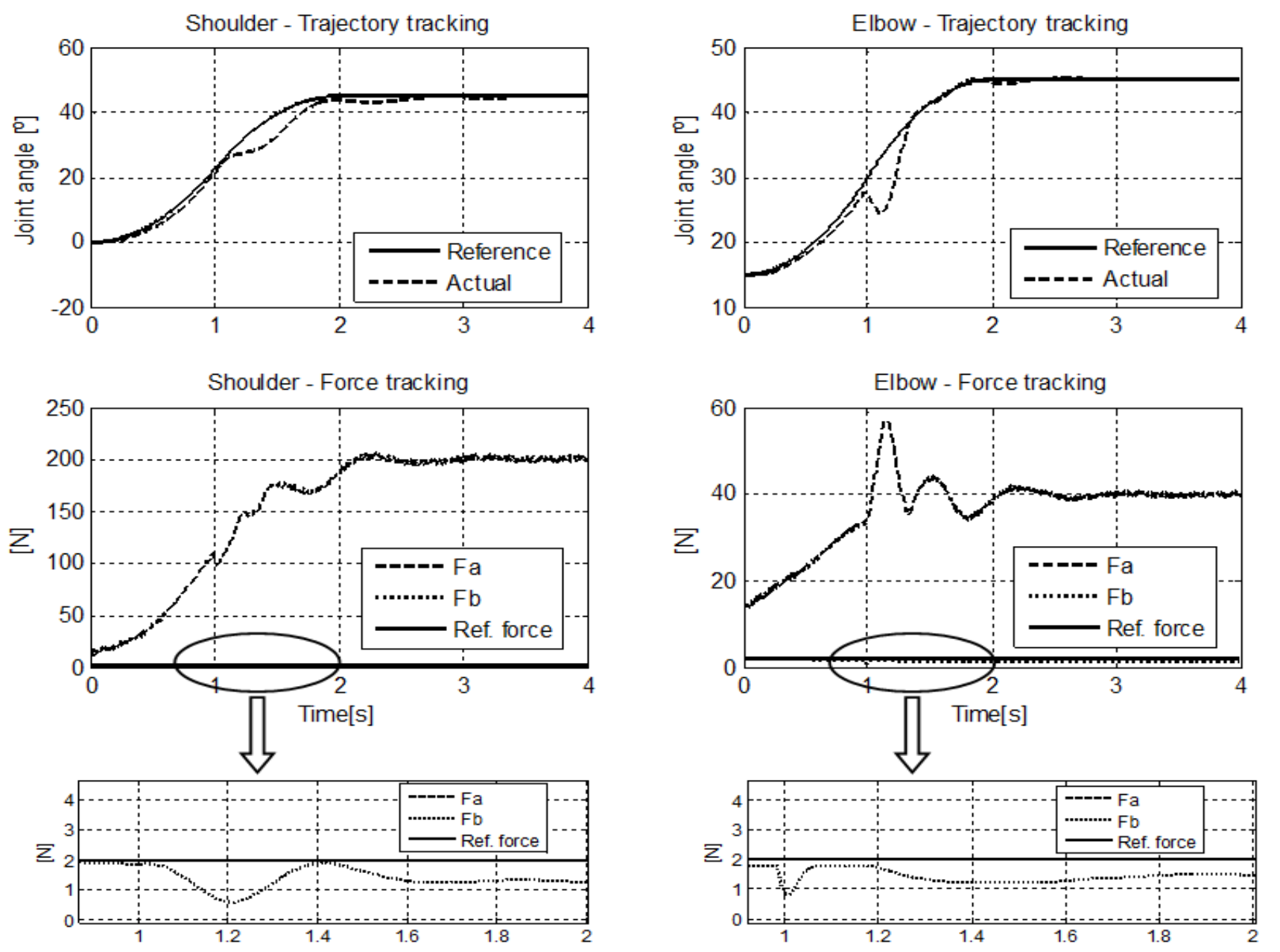

Fig 7. THE TRAJECTORY TRACKING AND THE FORCE TRACKING DURING THE GRASPING EXPERIMENT IN THE MULTI-JOINTED SYSTEM

body, the effective joint inertia and the gravity load depend on the entire system position, and the dynamic coupling between joints strongly disturbs the joint control. By applying robust control theory, the disturbances can be compensated, and the system works correctly, even in a demanding task such as grasping objects.

\section{ACKNOWLEDGMENTS}

The research leading to these results has received funding from the European Community's Seventh Framework Programme FP7/2007-2013 - Challenge 2- Cognitive Systems, Interaction, Robotics - under grant agreement no. 231864- ECCEROBOT; and partly by the Serbian Ministry of Science and Technological Development under contracts 35003 and 44008.

\section{REFERENCES}

[1] Fukuda T., Michelini R., Potkonjak V., Tzafestas S., Valavanis K., Vukobratovic M., 2001, "How Far Away is "Artificial Man"," IEEE Robotics and Automation Magazine, pp. 66-73.

[2] Potkonjak V., Svetozarevic B., Jovanovic K., Holland O., 2010, "Biologically-inspired Control of a Compliant Anthropomimetic Robot," The 15th IASTED International Conference on Robotics and Applications, The International Association of Science and Technology for Development, Cambridge, Massachusetts, pp. 182-189

[3] Potkonjak V., Vukobratovic M., 2005, "A Generalized Approach to Modeling Dynamics of Human and Humanoid Motion," Intl. Journal of Humanoid Robotics (World Scientific publ.), 2(1), pp. $1-24$.

[4] Potkonjak V., Vukobratovic M., Babkovic K., Borovac B., 2007, "Simulation Model of General Human and Humanoid Motion," 7Intl. J. Multibody System Dynamics (Springer), 17(1), pp. 7196. 
[5] Jacobsen S. C., Wood J. E., Knutti D. F., Biggers K. B., Iversen E. K., 1984, "The UTAH/M.1.T. Dextrous Hand: Work in Progress," The International Journal of Robotics Research, 4(3), pp. 21-50.

[6] Jacobsen S. C., Ko H., Iversen E. K., Davis C. C., 1990, "Control strategies for tendon-driven manipulators," IEE Control Systems Magazine, 10, pp. 23-28.

[7] Chang S. L., Lee J. J., Yen H. C., 2005, "Kinematic and compliance analysis for tendon-driven robotic mechanisms with flexible tendons," Mechanism and Machine Theory, 40, pp. 728739.

[8] Zinn M., Roth B., Khatib O., Salisbury J. K., 2004, “A new actuation approach for human friendly robot design," The International Journal of Robotics Research, 23, pp. 379-398.

[9] Klug S., Lens T., Von Stryk O., Mohl B., Karguth A., 2008, "Biologically Inspired Robot Manipulator for new Applications in Automation Engineering," Proceedings of Robotik, Munich.
[10] Potkonjak V., Svetozarevic B., Jovanovic K., Holland O., 2010, "Control of Compliant Anthropomimetic Robot Joint," International Conference of Numerical Analysis and Applied Mathematics, American Institute of Physics, Rhodes, pp. 1271-1274.

[11] Guyton A. C. and Hall J. E., 2006, Textbook of medical physiology, 11th Edition, Elsevier Saunders, Philadelphia.

[12] Khalil H. K., 2002, Nonlinear Systems, Third Edition, Prentice Hall, Upper Saddle River, New Jersey.

[13] McFarlane D. C. and Glover K., 1992, “A Loop Shaping Design Procedure using Synthesis," IEEE Transactions on Automatic Control, 37(6), pp. 759- 769. 